\title{
eLoran Signal Strength and Atmospheric Noise Simulation over Korea
}

Joon Hyo Rhee, Jiwon Seo ${ }^{\dagger}$

School of Integrated Technology, Yonsei University, Incheon 406-840, Korea

\begin{abstract}
GPS is the most widely-used Positioning, Navigation, and Timing (PNT) system. Since GPS is an important PNT infrastructure, the vulnerability of GPS to signal jamming has received significant attention. Especially, South Korea has experienced intentional high-power jamming from North Korea for the past three years, and thus realized the necessity of a complementary PNT system. South Korea recently decided to deploy a high-power terrestrial navigation system, eLoran, as a complementary PNT system. According to the plan, the initial operational capability of the Korean eLoran system is expected by 2016, and the full operational capability is expected by 2018. As a necessary research tool to support the Korean eLoran program, an eLoran performance simulation tool for Korea is under development. In this paper, the received signal strength, which is necessary to simulate eLoran performance, from the suggested Korean eLoran transmitters is simulated with the consideration of effective ground conductivities over Korea. Then, eLoran signal-to-noise ratios are also simulated based on atmospheric noise data over Korea. This basic simulation tool will be expanded to estimate the navigation performance (e.g., accuracy, integrity, continuity, and availability) of the Korean eLoran system.
\end{abstract}

Keywords: eLoran, SNR simulation, signal attenuation, effective ground conductivity, atmospheric noise

\section{INTRODUCTION}

GPS plays a critical role in the navigation systems of modern society. GPS is widely used in automotive navigation systems, scientific research, and surveying as well as the operations of airplanes and ships. It is also used in most mobile phones. Accordingly, the simple and convenient navigation and positioning service of GPS is playing an important role in our daily lives. However, as GPS has become increasingly important, the vulnerability of GPS has also become a critical issue. It is well known that even a small and inexpensive jammer can easily overwhelm GPS signals. In addition, a research team at the University of Texas recently demonstrated GPS spoofing for an Unmanned Aerial Vehicle (UAV) and a yacht. Carroll (2003) studied the vulnerability of GPS and the risks. Narins et al. (2010) mentioned that GPS is not a robust system and thus an Alternative Position, Navigation, and Timing (APNT) system is needed.

Received Sep 09, 2013 Revised Oct 16, 2013 Accepted Oct 28, 2013 †Corresponding Author

E-mail: jiwon.seo@yonsei.ac.kr

Tel: +82-32-749-5833 Fax: +82-32-818-5801
Especially, the risks are higher in South Korea because it is confronting North Korea. As shown in Table 1, North Korea has attempted GPS jamming for an increasing period each year, and caused GPS disruptions to the airplanes, ships, and cell towers in South Korea. The damage has gradually increased each year, and in 2012 for example, 1,016 airplanes and 254 ships could not receive GPS signals due to the 16-day jamming attack. Because of the seriousness of this problem, studies have been actively conducted regarding complementary Positioning, Navigation, and Timing (PNT) systems for the event of GPS outages.

As a complementary PNT system, South Korea has

Table 1. GPS disruptions in South Korea for the past three years due to North Korean jamming (Table. 1 of Seo \& Kim 2013).

\begin{tabular}{|c|c|c|c|}
\hline Dates & $\begin{array}{c}\text { Aug } 23-26 \\
2010 \\
\text { (4 days) }\end{array}$ & $\begin{array}{c}\text { Mar 4-14, } \\
2011 \\
\text { (11 days) } \\
\end{array}$ & $\begin{array}{c}\text { Apr 28-May 13, } \\
2012 \\
\text { (16 days) } \\
\end{array}$ \\
\hline Jammer locations & Kaesong & $\begin{array}{c}\text { Kaesong, } \\
\text { Mt. Kumgang }\end{array}$ & Kaesong \\
\hline Affected areas & $\begin{array}{l}\text { Gimpo, } \\
\text { Paju, etc. }\end{array}$ & $\begin{array}{l}\text { Gimpo, Paju, } \\
\text { Gangwon, etc. }\end{array}$ & $\begin{array}{l}\text { Gimpo, } \\
\text { Paju, etc. }\end{array}$ \\
\hline GPS disruptions & $\begin{array}{c}181 \text { cell towers, } \\
15 \text { airplanes, } \\
1 \text { battle ship }\end{array}$ & $\begin{array}{c}145 \text { cell towers, } \\
106 \text { airplanes, } \\
10 \text { ships }\end{array}$ & $\begin{array}{c}\text { 1,016 airplanes, } \\
254 \text { ships }\end{array}$ \\
\hline
\end{tabular}


recently decided to deploy enhanced LOng RAnge Navigation (eLoran) system (Seo \& Kim 2013). eLoran is a state-of-the-art terrestrial radionavigation system, which significantly improves the navigation performance such as accuracy, integrity, continuity, and availability of the previous Loran-C system. Since the output power of transmitting signals is very high and the frequency (100 $\mathrm{kHz}$ ) is low, it is virtually impossible to jam eLoran signals over a wide area using a small antenna and jammer. Therefore, even when GPS is unavailable due to jamming, eLoran can continuously provide PNT service. Because of its clear benefit, other countries such as the United Kingdom are also considering to deploy eLoran.

The United Kingdom (UK) has been operating a prototype eLoran system by upgrading the Anthorn Loran-C station to an eLoran station (Johnson et al. 2007). In addition, it was recently announced that seven differential eLoran stations will be installed in the UK. The UK is planning to use these stations to support harbor entrance and approach of ships along the South and East coast of the UK when GPS is unavailable.

South Korea is planning to install and operate five eLoran transmitters and 43 differential eLoran stations so that eLoran service can be provided to the entire country (Seo \& Kim 2013). The eLoran transmitters in Korea aim to begin initial operation from 2016, continue test operation for 2 years, and reach Full Operational Capability (FOC) in 2018. According to the plan, Korea will be the first nation to provide eLoran service to the entire country.

With the deployment of the eLoran system in Korea, a performance simulation tool, which can analyze, model, and predict the performance of the system depending on various situations and environments, is also needed. A simulation tool can predict the performance of various integrity algorithms of eLoran receivers as well as can simulate the navigation performance of the eLoran system. Thus, a performance simulation tool will be useful for the operation and performance improvement of the eLoran system.

Several eLoran performance simulation tools have been previously developed. The Global Lighthouse Authorities of the United Kingdom and Ireland (GLA) have developed a simulation software named BALOR, which can simulate eLoran accuracy and coverage by predicting Additional Secondary Factor (ASF) that is the major error factor of an eLoran system (Williams \& Last 2004). Also, Stanford University has developed a simulation tool, which can predict the integrity, continuity, and availability of an eLoran system as well as the accuracy (Lo et al. 2008).

To predict and simulate the performance of an eLoran system, the signal propagation environment of the region and its effects need to be studied. As eLoran signals are groundwaves that are transmitted along the Earth's surface, the propagation delays of eLoran signals are closely related to the characteristics of the Earth's surface. The representative characteristics of the Earth's surface, which affect the signals, include terrain, altitude, and effective ground conductivity. Regarding the effects of the Earth's surface on eLoran signals, Monteath et al. (1978) suggested a representative model for calculating the propagation delays of groundwaves in irregular terrain, and Last et al. (2000) studied the modeling of signal propagation in irregular terrain. In addition, the International Telecommunication Union Radiocommunication Sector (ITU-R) measured the effective ground conductivity of the Earth's surface across the globe and performed the documentation of the obtained data (ITU-R P.832.3 1992), and also studied the relation between effective ground conductivity and signal attenuation at various frequencies (ITU-R P.368-7 1992).

Moreover, the atmosphere as well as the Earth's surface affects eLoran signals. Feldman (1972) and Cutright et al. (2005) studied the relation between atmospheric noise and Loran-C signals, and Sammaddar (1980) analyzed the effects of weather on propagation delays. Also, Boyce (2007) studied atmospheric noise and the corresponding Signalto-Noise Ratios (SNR) of eLoran signals.

In this paper, based on these previous studies, the expected SNR of eLoran signals over Korea is analyzed. First, the received signal strengths of eLoran signals are simulated considering the effects of effective ground conductivity on the attenuation of eLoran signals. Using this result and the atmospheric noise data, eLoran SNR over Korea is estimated and presented on a map.

In Section 2, the general background of an eLoran system is summarized; and in Section 3, the characteristics of effective ground conductivity and atmospheric noise, which are the two environmental factors considered in this study, are explained. In Section 4, eLoran SNR over Korea is calculated using effective ground conductivity and atmospheric noise, and the result is presented on a map. Conclusions are given in Section 5.

\section{BACKGROUND}

eLoran is a terrestrial radionavigation system using groundwaves, which upgrades the previous Loran-C system and significantly improves its navigation performance such as accuracy, integrity, continuity, and availability. The Loran-C systems, which provide an accuracy of about 
Table 2. Requirements for harbor operations and aviation (Lo 2008).

\begin{tabular}{ccccc}
\hline Requirements & Accuracy & Integrity & Availability & Continuity \\
\hline $\begin{array}{c}\text { HEA } \\
\text { equirements }\end{array}$ & $20 \mathrm{~m}, 2 \mathrm{drms}$ & $3 \times 10^{-5} /$ hour & $99.7 \%$ & $\begin{array}{c}99.85 \% \text { over } \\
3 \text { hours }\end{array}$ \\
$\begin{array}{c}\text { NPA } \\
\text { equirements }\end{array}$ & $307 \mathrm{~m}$ & $10^{-7} /$ hour & 99.9 to $99.99 \%$ & 99.9 to $99.99 \%$ \\
\hline
\end{tabular}

$460 \mathrm{~m}$, have not been widely used after the development of GPS. However, eLoran increases its accuracy up to 8 20 $m$ by sending differential corrections through eLoran Data Channel (LDC) that was not present in Loran-C. Unlike Loran-C signals, eLoran signals are synchronized to Coordinated Universal Time (UTC). Thus, eLoran receivers can calculate position solutions based on the Time of Arrival (TOA) of all received signals regardless of the chains of transmitters, which is very similar to the way of calculating positions by GPS receivers. On the other hand, Loran-C receivers rely on the Time Difference of Arrival (TDOA) of the signals from the same transmitter chain for position calculation. Hence, the availability of Loran-C is generally lower than the availability of eLoran.

Table 2 summarizes the performance requirements of navigation systems for harbor operations and aviation. The Harbor Entrance and Approach (HEA) requirements are considered for ships to approach harbors, and the NonPrecision Approach (NPA) requirements are considered for airplanes to approach airports. According to the International Loran Association (2007), an eLoran system can meet the above two requirements, and thus eLoran is expected to be widely used in harbor operations and aviation. In addition, eLoran can also be used for land surface navigation and indoor applications.

In the case of Korea, five transmitter stations are planned to be constructed in Pohang, Kwangju, Jeju, Ganghwa, and Ulleungdo, as shown in Fig. 1. For the five transmitters, specifications such as transmitting power, antenna height, etc. have already been determined, and the sites are secured. In addition, to obtain high navigation performance on the land as well as in the air and on the sea, 43 differential eLoran stations are planned to be installed in regions including inland areas. As mentioned in Section 1, Initial Operational Capability (IOC) is planned to be announced in 2016 and FOC in 2018 after two years of test operations.

\section{ELORAN SIGNAL PROPAGATION ENVIRONMENTS}

\subsection{Effective Ground Conductivity}

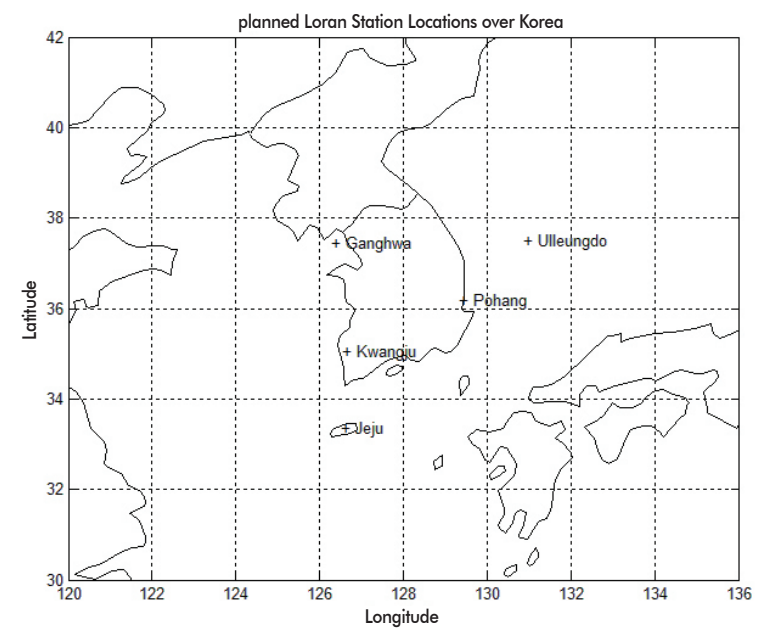

Fig. 1. Locations of the planned Korean eLoran transmitters.

When typical radio waves propagate, signal power attenuates logarithmically depending on distance. Thus, if the distance between a user and a transmitter is known, the approximate strength of radio waves received by the user can be estimated using this distance information. However, in practice, the strength cannot be obtained accurately using the distance information alone, but various other propagation environment factors need to be considered.

eLoran signals are groundwaves that propagate along the Earth's surface. For groundwaves, one of the major factors that affect the propagation is effective ground conductivity. The effective ground conductivity represents the conductivity of the Earth's surface that affects the propagation of radio waves, and it is determined by the condition and characteristics of the Earth's surface. The effective ground conductivity is different from the ground conductivity that is directly measured for the soil of the Earth's surface. The effective ground conductivity is a value that quantifies the effects of the Earth's surface on groundwaves. Table 3 summarizes approximate effective ground conductivities and permittivities of various types of terrains. In general, the effective ground conductivity of the sea is $5 \mathrm{~S} / \mathrm{m}$, and the effective ground conductivity of the land is $1 \sim 8 \mathrm{mS} / \mathrm{m}$ depending on the characteristics of the Earth's surface. ITU measured and documented the effective ground conductivity of the entire globe for the radio waves in Medium Frequency (MF) and Low Frequency (LF). The effective ground conductivity over Korea was also measured, and the results were presented in ITU-R P.832.3 (1992), as shown in Fig. 2.

For the signal strength attenuation model based on effective ground conductivity, ITU developed a software called 'GRWAVE' and constructed the database (Whitington \& Thomas 1992). In our paper, the attenuation of signals depending on effective ground conductivity is calculated using this model developed by ITU. For example, the attenuations of signal strength due to various effective ground conductivities can be calculated as in Fig. 3, when the signals with 
Table 3. Effective ground conductivities and permittivities of various types of terrains (Table 2.1-2 of Barringer \& Springer 1992).

\begin{tabular}{ccc}
\hline Types of terrain & $\begin{array}{c}\text { Effective ground } \\
\text { conductivity (S/m) }\end{array}$ & $\begin{array}{c}\text { Relative Dielectric } \\
\text { Constant, (esu) }\end{array}$ \\
\hline Seawater & 5 & 80 \\
Fresh Water & $8 \times 10^{-3}$ & 80 \\
Dry Sandy, flat coastal land & $8 \times 10^{-3}$ & 10 \\
Marshy, forested flat land & $8 \times 10^{-3}$ & 12 \\
Rich agricultural land, low hills & $1 \times 10^{-2}$ & 15 \\
Pasture land, medium hills and forest & $5 \times 10^{-3}$ & 13 \\
Rocky land, steep hills & $2 \times 10^{-3}$ & 10 \\
Mountainous & $1 \times 10^{-3}$ & 5 \\
Residential Area & $2 \times 10^{-3}$ & 5 \\
Industrial Area & $1 \times 10^{-4}$ & 3 \\
\hline
\end{tabular}

transmitting power of $1000 \mathrm{~kW}$ are received by an antenna with $3 \mathrm{~dB}$ gain. This calculation is based on the data of ITUR P.368.7 (1992).

If the propagation path of radio waves consists of a single terrain type, signals attenuate following the curves shown in Fig. 3. However, if the propagation path consists of various terrain types, signal attenuation would not follow the curve because effective ground conductivity differs depending on the types of terrains. In this case, the propagation path can be divided into small intervals, and attenuation is separately calculated in each interval using the effective ground conductivity of the interval. Then, the total attenuation is obtained by integrating the attenuations of all small intervals. The calculated total attenuation is used to predict the received signal strength at an arbitrary location.

\subsection{Atmospheric Noise}

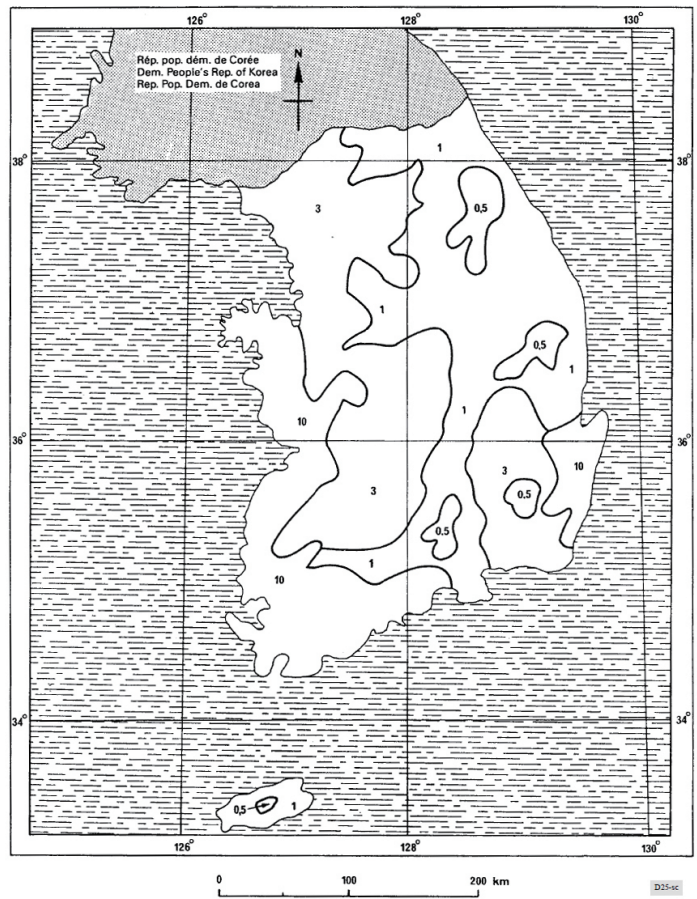

Fig. 2. Effective ground conductivity data over Korea (Fig. 25 of ITU-R P.832.3, 1992)

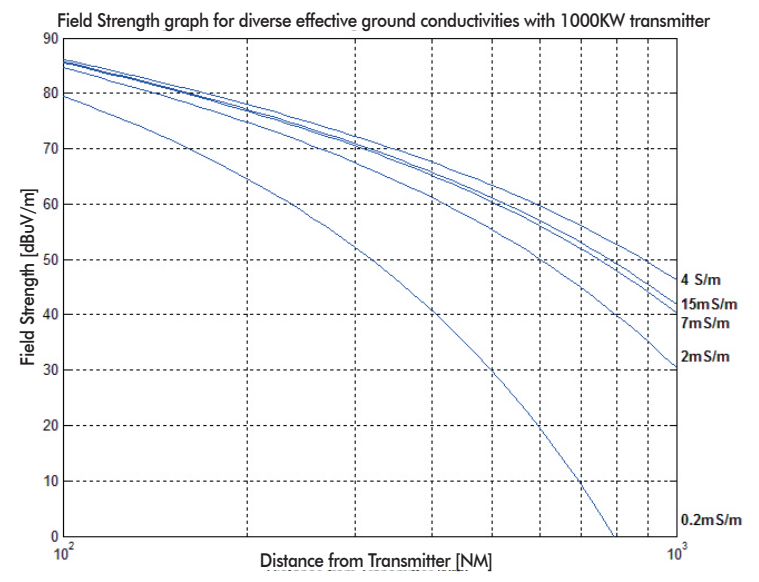

Fig. 3. Signal strength in $\mathrm{dB}$ vs. distance graph for various effective ground conductivities (based on the data from ITU-R P.368-7, 1992).

Among various studies on atmospheric noise in the literature, Boyce's work is the most relevant to eLoran. Boyce (2007) studied the characteristics of atmospheric noise, calculated SNR between Loran signals and atmospheric noise, and developed new atmospheric noise models. In this section, a part of the atmospheric noise study of Boyce (2007) is introduced, and atmospheric noise data over Korea is calculated and presented on a map.

Atmospheric noise refers to the noise which occurs due to the effects of the atmosphere along the path where radio waves propagate, and it is mostly generated by electric discharge (i.e., lightning). According to Boyce (2007), the types of lightning can be classified into cloud-to-ground (C2G) lightning and ground-to-cloud (G2C) lightning, which account for about $40 \%$ of total lightning, and intracloud (IC) lightning, which accounts for about $50 \%$. The rest are cloudto-cloud (C2C) lightning and cloud-to-air (C2A) lightning, which account for about $10 \%$. Lightning causes electric noise, which occurs in the frequency bands between $1 \mathrm{kHz}$ and $20 \mathrm{MHz}$, and has a peak near the $10 \mathrm{kHz}$ frequency. CCIR, which is the former ITU, measured the atmospheric noise of low frequency radio wave communication across the globe from 1956 to 1970 . The results were summarized in the CCIR-322-3 document, which was completed in 1986 after the modification of the model and the update of the data. In 1992, CCIR was reorganized as ITU, and the document was redistributed as the ITU-R P.372-7 document through the additional modification and the insertion of the expanded data.

ITU calculated a statistic called 'external antenna noise factor $\left(F_{a}\right)$ ' based on the noise data which had been measured for several years. $F_{a}$ represents the power that is received by a loss-free antenna in specific season and at specific time. $F_{a}$ is a value that varies depending on season and time slot. ITU calculated the $F_{a}$ values of the entire globe through the interpolation of the obtained values, and constructed the database for each season and time slot. $F_{a}$ is a random variable that follows log-normal distribution rather 
(a)
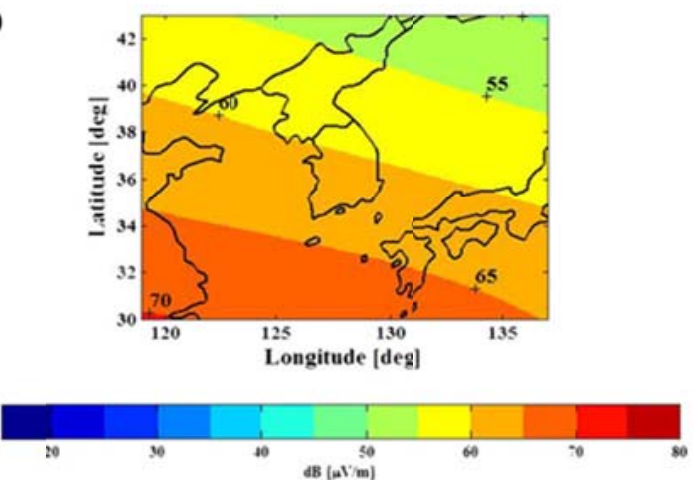

(c)
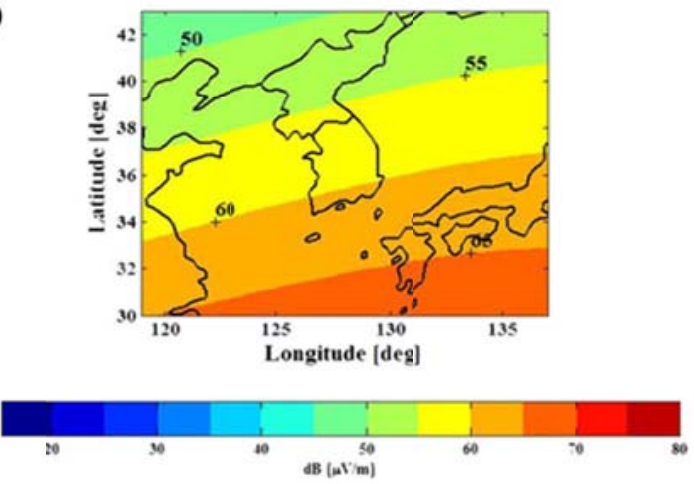

(b)
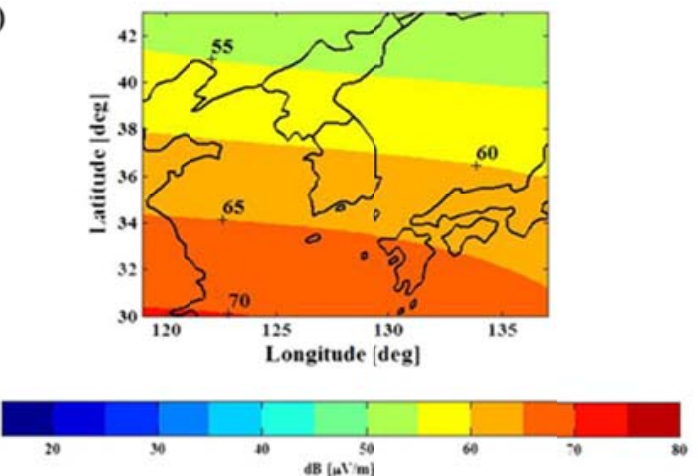

(d)

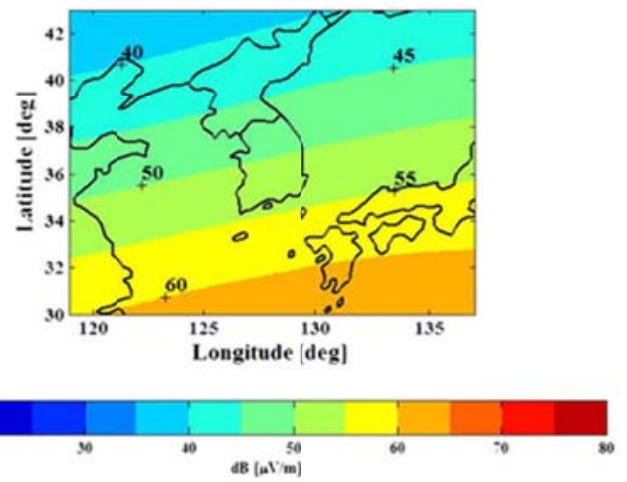

Fig. 4. (a) Atmospheric noise $E_{n}$ over Korea during 17:00 18:00 in spring. (b) Atmospheric noise $E_{n}$ over Korea during 17:00 18:00 in summer. (c) Atmospheric noise $E_{n}$ over Korea during 17:00 18:00 in autumn. (d) Atmospheric noise $E_{n}$ over Korea during 17:00 18:00 in winter .

than a fixed constant. As a representative value, ITU calculated an average value based on $90 \% F_{a}$ data. To convert this into $F_{a}$ at $P \%$ condition, Eq. (1) is used.

$F_{a, P}=F_{a m}+D_{u} \cdot \operatorname{norminv}(P / 100,0,1) / \operatorname{norminv}(90 / 100,0,1)$

where $F_{a m}$ represents the average value of the random variable $\left(F_{a}\right)$, and $D_{u}$ represents the upper decile of the probability distribution. The obtained $F_{a, P}$ can be converted into $E_{n}$ using Eq. (2), as derived in Boyce (2007). $E_{n}$ represents the rms envelope noise voltage.

$E_{n}[\mathrm{~dB}(\mu \mathrm{V} / \mathrm{m})]=F_{a, P}+20 \log _{10} f_{M H z}+10 \log _{10} b-95.5$

where $f_{M H z}$ represents the frequency expressed in $\mathrm{MHz}$, and $b$ represents the bandwidth of the noise power.

Based on this equation, the atmospheric noise $\left(E_{n}\right)$ at a specific location for each season and time slot can be calculated in $\mathrm{dB}(\mu \mathrm{V} / \mathrm{m})$. If $E_{n}$ is calculated from the $F_{a}$ data over Korea, the atmospheric noise for each season and time slot for Korea can be obtained. For example, Fig. 4 shows the $E_{n}$ of Korea for 17:00 18:00 time slot in each season assuming the $95 \% F_{a}$. In general, the atmospheric noise is larger in summer than in winter, and is larger during the day than during the night. Also, the atmospheric noise shows increasing trend as latitude decreases. The quality of received
eLoran signals is affected by the ratio between eLoran signals and atmospheric noise.

\section{SNR SIMULATION OF THE KOREAN ELORAN SYSTEM}

\subsection{Received Signal Strength Simulation}

The attenuation of radio signals can be estimated based on the log-distance path loss model. The received strength of radio signals decreases logarithmically in proportion to distance. Considering additional attenuation depending on effective ground conductivity, the attenuation of the signal strength of groundwaves can be expressed by Eq. (3).

$$
P L(r)[d B]=P L\left(r_{0}\right)-10 n \log \left(\frac{r}{r_{0}}\right)+e a
$$

$P L(r)$ represents the signal loss depending on distance $(r), r_{0}$ represents the reference distance, $n$ represents the propagation path loss factor, and ea represents the attenuation depending on effective ground conductivity. As mentioned in Section 3.1, the total attenuation depending on effective ground conductivity is obtained by integrating the attenuations of small distance intervals. Once the signal attenuation is calculated, the received signal strength can 
also be calculated.

Fig. 5 shows the simulation results of the received signal strength for the planned Pohang and Jeju eLoran transmitters of Korea. The transmitting power is assumed to be $1000 \mathrm{~kW}$, which is the currently planned value by the Korean government. The results indicate that eLoran signals would maintain above $60 \mathrm{~dB}$ for most of the country. However, signal strength alone cannot explain whether a receiver can actually calculate position solutions using the corresponding signals. It is because a receiver can perform signal tracking and position calculation when SNR, rather than the signal strength itself, is above a certain threshold. Therefore, in order to determine the eLoran coverage, SNR needs to be estimated. The SNR simulation requires the knowledge of atmospheric noise in addition to the received signal strength.

\subsection{SNR Simulation}

The SNR of eLoran in this paper refers to the ratio between the received signal strength calculated in Section 4.1 and the atmospheric noise calculated in Section 3.2. In general, a receiver can track eLoran signal if its SNR is above a certain threshold. Thus, eLoran service coverage is related to the SNR and the tracking threshold of a receiver. In this paper, the eLoran SNR is simulated and analyzed over Korea with the consideration of its propagation environment and expected transmitter locations and powers.

Fig. 6 shows the predicted SNR over Korea in spring for the signals from the Pohang, Kwangju, Jeju, Ganghwa, and Ulleungdo transmitters. For this simulation, the $F_{a}$ is assumed to be $95 \%$, the season is set to spring, and the time slot is selected to be the one with the largest atmospheric noise of a day, as an example. The obtained SNR in this paper considers the received signal strength just before the front-end of a receiver. The actual tracking performance of a specific receiver also depends on its internal noise, processing gain, tracking algorithm, etc. However, these characteristics of a receiver could significantly vary among different receivers. Therefore, the SNR before the front-end is analyzed in this study so that the result can be applied to any receivers once receiver-specific parameters are further considered.

\section{CONCLUSIONS}

South Korea realized the importance of a complementary PNT system to backup GPS and recently decided to deploy eLoran system. In order to estimate the navigation performance of eLoran such as accuracy, integrity, continuity, and availability, the SNR of eLoran signals over Korea needs to be calculated. In this paper, effective ground conductivity and atmospheric noise are considered as the major parameters affecting the propagation of eLoran signals. The received signal strength is simulated by considering the effects of effective ground conductivity on the attenuation of eLoran signals, and the atmospheric noise $\left(E_{n}\right)$ over Korea is estimated using the data measured by ITU. Based on these results considering propagation environments of Korea, the eLoran SNR over Korea is simulated. This SNR simulation is a basis of developing an eLoran performance simulation tool for Korea which is a topic of future studies.

\section{ACKNOWLEDGMENTS}

The authors gratefully acknowledge Sherman Lo, Stanford University, for the valuable comments and information for this study. This research was a part of the project titled "Developing eLoran Coverage and Performance Simulation Tool for Korea" funded by the Ministry of Oceans and Fisheries, Korea. This research was also supported by the Ministry of Science, ICT and Future Planning (MSIP), Korea, under the "IT Consilience Creative Program" (NIPA2013-H0203-13-1002) supervised by the National IT
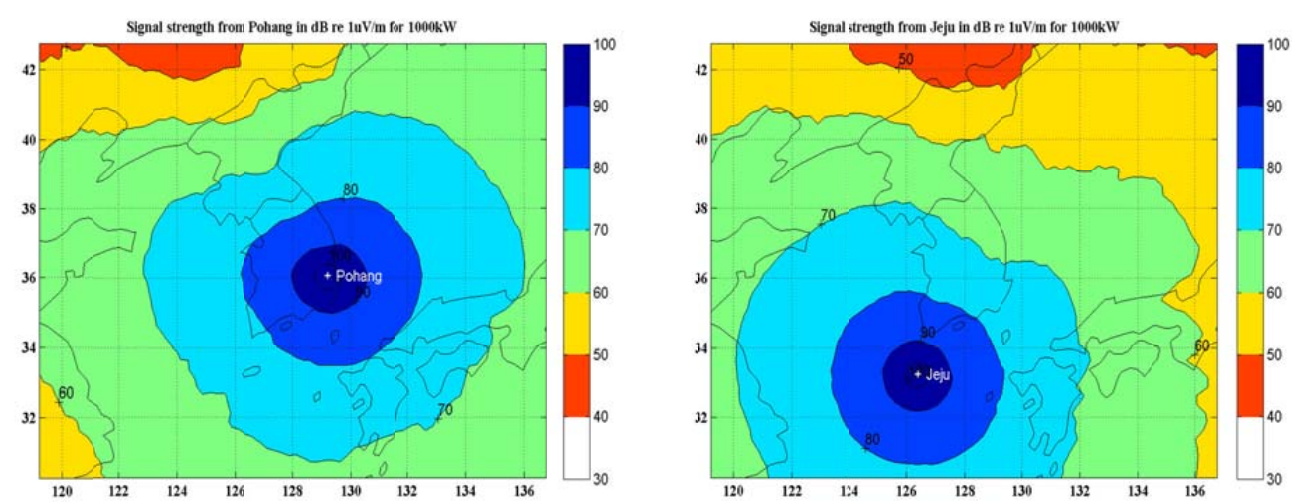

Fig. 5. eLoran signal strength simulation for the planned Pohang and Jeju transmitters. 
(a)

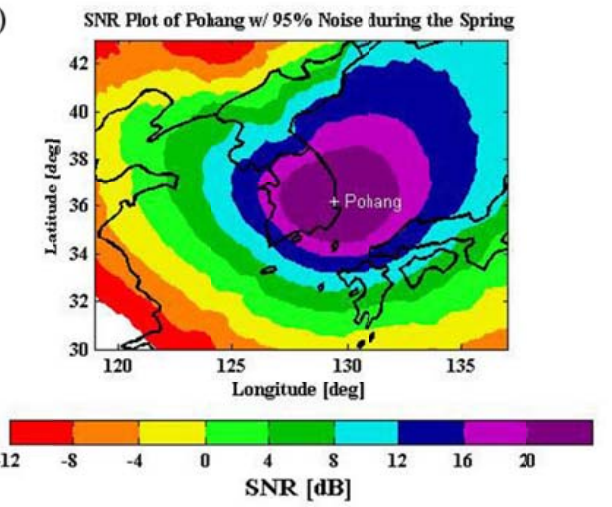

(c)

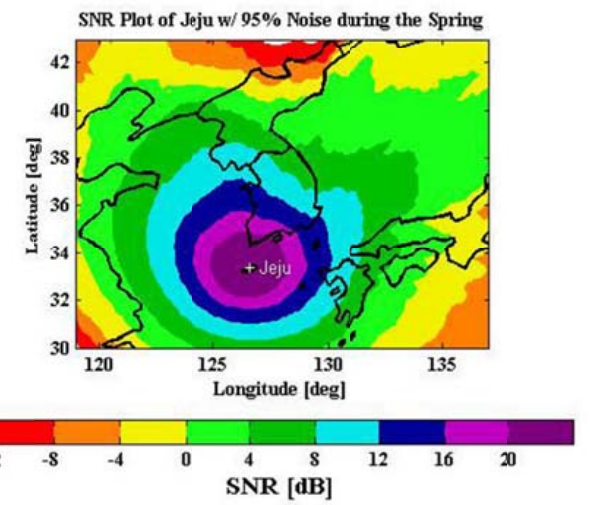

(e)

SNR Plot of Ulleungdo w/ $95 \%$ Noise during the Spring

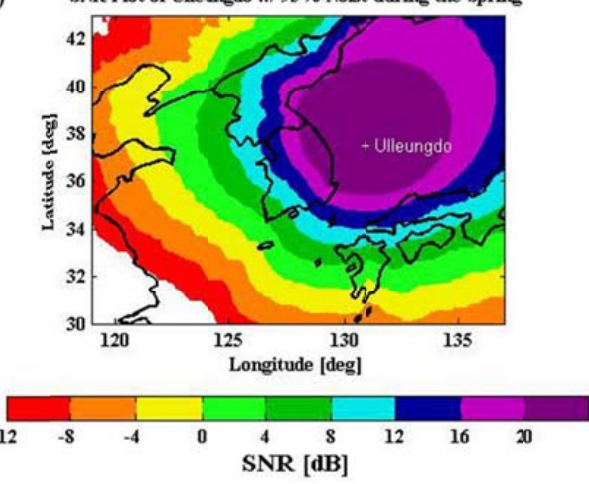

(b)

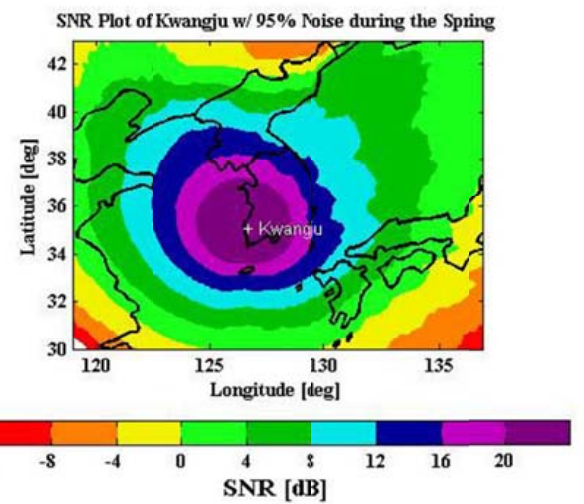

(d)

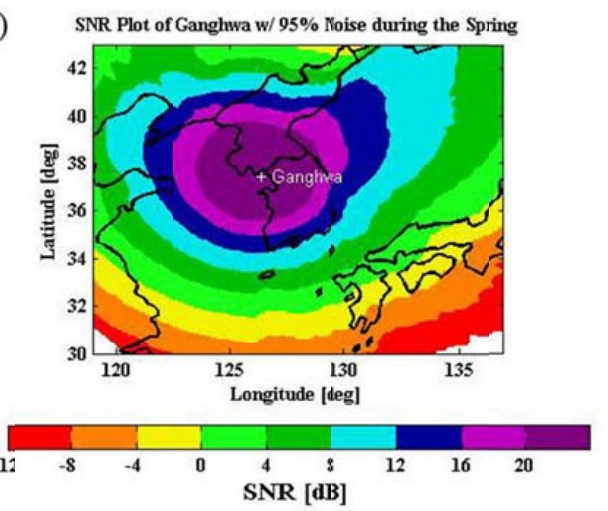

Fig. 6. (a) eLoran SNR simulation for the signal of Pohang transmitter in spring. (b) eLoran SNR simulation for the signal of Kwangju transmitter in spring. (c) eLoran SNR simulation for the signal of Jeju transmitter in spring. (d) eLoran SNR simulation for the signal of Ganghwa transmitter in spring. (e) eLoran SNR simulation for the signal of Ulleungdo transmitter in spring.

Industry Promotion Agency (NIPA).

\section{REFERENCES}

Barringer, M. H. \& Springer, K. D. 1992, Radio wave propagation, NAB Eng. Handbook, Washington, DC: NAB, ch. 2.1.

Boyce Jr., C. O. L. 2007, Atmospheric Noise Mitigation for Loran, Ph.D. dissertation, Stanford University, Stanford, CA, June 2007

Carroll, J. V. 2003, Vulnerability assessment of the US transportation infrastructure that relies on the Global Positioning System, NAVIGATION: The Journal of Navigation, Vol. 56, No. 2, pp. 185-193.

Cutright, C., Sayre, J., \& van Graas, F. 2005, Analysis of the Effects of Atmospheric Noise on LORAN-C, Proceedings of the International LORAN Association (ILA-34) Convention and Technical Symposium, Santa Barbara, CA, October 17-19, 2005

Feldman, D. A. 1972, An Atmospheric Noise model with Application to Low Frequency Navigation Systems, Ph.D. thesis, Massachusetts Institute of Technology, Boston, MA, June 1972 
International Loran Association, 2007, eLoran Definition Document, www.loran.org

International Telecommunication Union Radiocommunication Sector, 1992, Groundwave Propagation Curves for Frequencies between $10 \mathrm{kHz}$ and $30 \mathrm{MHz}$, REC.ITU-R P.368-7

International Telecommunication Union Radiocommunication Sector, 1992, World Atlas of Ground Conductivities, REC.ITU-R P.832.3

Johnson, G. W., Dykstra, K., Oates, C., Swaszek, P. F., \& Hartnett, R. 2007, Navigating Harbors at High Accuracy Without GPS: eLoran Proof-of-Concept on the Thames River, Proceedings of the 2007 National Technical Meeting of The Institute of Navigation, San Diego, CA, USA, January 2007, pp. 1201-1211

Last, J. D., Williams, P., Peterson, B. B., \& Dykstra, K. 2000, Propagation of Loran-C Signals in Irregular Terrain - Modelling and Measurements Part 1: Modelling, 29th Annual Convention and Technical Symposium, International Loran Association, Washington DC, Washington, USA, 13-15 November 2000

Lo, S. C., Peterson, B. B., Boyce Jr., C. O. L., \& Enge, P. K. 2008, Loran Coverage Availability Simulation Tool, Proceedings of the Royal Institute of Navigation NAV08 / International Loran Association 37th Annual Meeting

Monteath, G. D. 1978, Computation of Groundwave Attenuation over Irregular and Inhomogeneous Ground at Low and Medium Frequencies, BBC Report 1978/7, British Broadcasting Corporation, Research and Development, Kingswood Warren, Tadworth, Surrey, UK

Narins, M., Eldredge, L., Enge, P. K., Harrison, M., Kenagy, R., \& Lo, S. 2010, Alternative Position, Navigation, and Timing-The Need for Robust Radionavigation, Royal Institute of Navigation -NAV 10, London, UK

Sammaddar, S. N. 1980, Weather Effect on Loran-C Propagation. Navigation, Journal of The Institute of Navigation, Vol. 27, No. 1, pp. 39-53, Washington, D.C.

Seo, J. \& Kim, M. 2013, eLoran in Korea - Current Status and Future Plans, Proceedings of the European Navigation Conference 2013, 23-25 April 2013, Vienna, Austria

Whitington, M. J. \& Thomas, R. M. 1992, Software for Prediction and Analysis of Ground Wave Propagation Loss, Surveillance Research Laboratory, Salisbury, South Australia

Williams, P. \& Last, J. D. 2004, Extending the Range of Loran-C ASF Modeling, International Loran Association, Tokyo, Japan, 26 October 2004

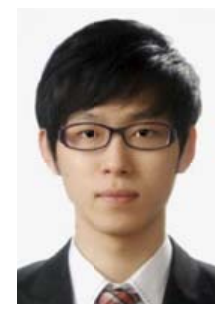

Joon Hyo Rhee is an M.S./Ph.D. student in the School of Integrated Technology, Yonsei University, Korea. He received his B.S. degree in electrical and electronic engineering from Yonsei University in 2012. His current research focuses on complementary navigation systems such as eLoran and intelligent unmanned systems. Mr. Rhee is a recipient of the Graduate Fellowship from the IT Consilience Creative Program supported by the Ministry of Science, ICT and Future Planning (MSIP), Korea.

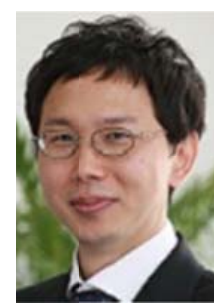

Jiwon Seo received his B.S. degree in mechanical engineering (division of aerospace engineering) from KAIST (Korea Advanced Institute of Science and Technology) and received M.S. degrees in aeronautics/astronautics and electrical engineering from Stanford University, Stanford, CA, and a Ph.D. degree in aeronautics/astronautics from Stanford University in 2010. He is currently an Assistant Professor in the School of Integrated Technology at Yonsei University, Korea. His research interests include alternative positioning, navigation, and timing systems such as eLoran; GPSbased intelligent transportation systems; and intelligent unmanned systems. Professor Seo is a member of the eLoran Advisory Committee of the Ministry of Oceans and Fisheries, Korea. 